\title{
Optimization of boiler's convection tubes based on Genetic Algorithm
}

\author{
Tianyu Zhang ${ }^{1,{ }^{*}}$, Qingfeng Zhang ${ }^{1}$, Zhenning Zhao $^{1}$, Liang Cheng $^{1}$, Gaojun Liu ${ }^{1}$ \\ ${ }^{1}$ North China Electric Power Research Institute, Beijing 100032, China; \\ *Corresponding author Email:azzztttyyy456@126.com
}

Keywords: Genetic Algorithm, convection tube, optimization, binary code.

\begin{abstract}
Genetic Algorithm (GA) is created by Prof. John Holland from Michigan University, deriving from the Darwin's theory of evolution, Weizmann's theory of species selection, and Mendel's theory of inheritance. In present study, the Genetic Algorithm is introduced to optimize the boiler convection tube bank of SHL32-2.5/AI and the method of programing group for optimization binary code,is achieved. Constraints are considered by means of penalty functions. Based on the data obtained in our case, the result shows in the almost equal amount of heat absorbed, the structure with optimization can economize on steel by $10 \%$. The idea of present study can also applied in economizer and other heat transfer array of tubes and can be used as a reference for further study.
\end{abstract}

\section{Introduction}

Genetic Algorithm (GA) is created by Prof. John Holland from Michigan University, deriving from the Darwin's theory of evolution, Weizmann's theory of species selection, and Mendel's theory of inheritance[1]. Its main idea lies on the simulation of mechanism of inherence and theory of evolution to search for optimization[2-4]. Up to now, it has been widely applied in automatic control, computation science, engineering design, intelligential fault diagnosis, management science and social science. GA is suitable for complex non-linear and multi-dimensional problems.

GA is an optimization method based on the natural species selection and ideas of evolution, and during the optimization period, the main character is that it will keep the crowd evolution. In case of the individual loses some useful information, the feature can be also delivered by other individuals.GA only need to have the information of objective function, and it doesn't require the questions to be differentiable which makes it widely suitable[1,5,6]. Meanwhile, it's an enlightening intelligential optimization method, so it can achieve better result in terms of highly complicated and multi-dimensional problems.

The procedure of optimization in GA begins with a production of random solutions, which is called as group. Every individual in the group is a solution to the problem, with the name of gene. These genes will evolve gradually in the sequent iteration, and the procedure of this kind of iteration is called inherence. The GA mainly produces the next generation by crossing, mutation and picking method, which is called posterity. The suitability of each gene is evaluated by the function derived from objective function. According the ranking of suitability, certain amount of genes will be chosen to be the group of next generation. Then after certain several iterations, the algorithms will convergence to the best gene, which is the most promising or less promising solution to the problem. Hence, the concept of suitability is utilized in GA to asset the degree a gene is approximating to the best solution. The criteria or the definition of the suitability is related with specific problem [7-10].

\section{Optimization analysis of boiler convection tubes}

\subsection{Validation of GA in optimization.}

In order to verify the ability of optimizing, GA is applied in the optimizing case of Shubert function.

Function of Shubert:

$$
F(x 1, x 2)=\left(\sum_{i=1}^{5} i \cdot \cos (i+1) \cdot x 1+i\right) \times\left(\sum_{i=1}^{5} i \cdot \cos (i+1) \cdot x 2+i\right)
$$


Set the range of $x 1, x 2$ as $[-10,10]$, the plot of the function is shown as below.

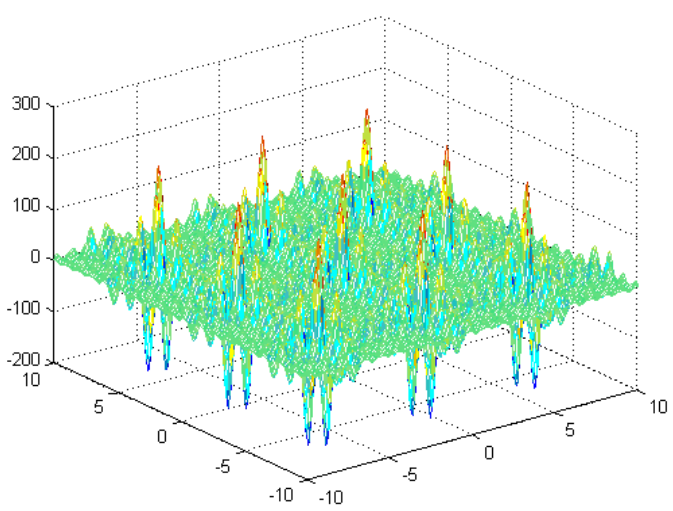

Fig.1 Plot of Shubert function

It can be seen that the function of Shubert is of multi-variable and multi-peak. Apply GA to optimize Shubert function, and results are obtained:

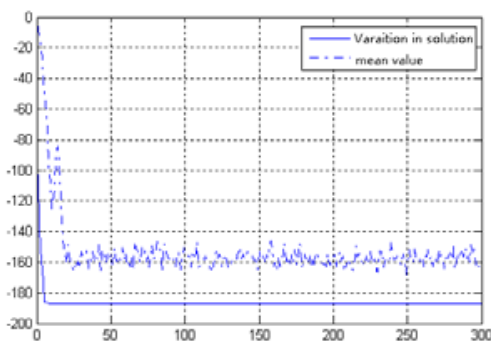

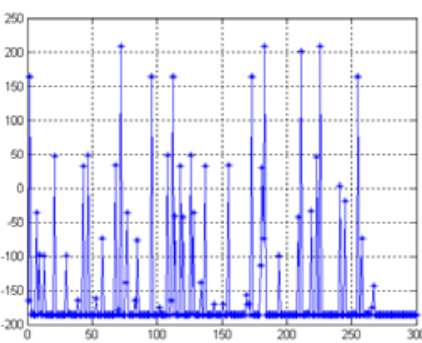

Fig.2 Variation of minimum and average value Fig.3 Distribution of values of 300 individuals As shown below, GA is proved to be compenent on convergence and global opitimization.

\subsection{Establishment of mathematical model based on GA.}

Convection tubes are main part to achieve heat transfer in boilers, with $40 \%$ steel consumption of the whole boiler. Therefore, the optimization design of convection tubes is of great importance to decrease the cost under the premise of guarantee the performance of heat transfer. The following part is to introduce the configuration optimization of the convection tubes from the boiler SHL35-2.5/AI.

Heat transfer formula of convection surface:

$$
Q=k \times \text { deltat } \times A
$$

Where $K$ is heat transfer coefficent $W /\left(m^{2} \times K\right)$, deltat is mean difference in temperature, $A$ is area of heat transfer $m^{2}$.

According to the formula (2), when the temperatures of the flue gas in the inlet and outlet remain constant, and the amount and temperature difference of heat transfer is fixed, the only way to decrease area is to increase the heat transfer conefficient.

The area of convection tubes is calculated as following:

$$
A=p i \times d \times L \times z 1 \times \mathrm{z} 2
$$

Where, $d$ is outer diameter of the tube, $z 1, z 2$ are the number of tubes along the row and column, respectively, the length of elbows of the tubes is counted into $L$.

The heat transfer coefficient $K$ of convection tubes in staggered tubes:

$$
K=f(K c, K r, f i)
$$

Where, $K$ is heat transfer coefficient, $K r$ is the heat transfer coefficient of thermal radiation, $f i$ is the efficient factor:

$$
\begin{gathered}
K c=f(d, w, s 1, r H 2 O, z 2, \text { tav }) \\
K r=f(t w a l, \text { tav, } r H 2 O, r t r i, d, s 1, s)
\end{gathered}
$$


Where s1,s2 are the intervals of the tubes along the row and column, respectively, twal is the temperature of wall covered with ash.

$$
\begin{gathered}
w=f(\text { Bcal }, V g, \text { tav }, f 1) \\
f 1=f(L, W, z 1, d)
\end{gathered}
$$

where, $f 1$ is the average area of glue gas, $W$ is the width of the furnace, $B c a l$ is the calculated fuel, $V g$ is the volmn of the flue gas.

As seen from above equations, the heat transfer coefficient $K$ is coupled with many factors. Based on the designed condition of boiler SHL35-2.5/AI, some factors can be set in advance:

Then $K$ can be expressed as:

$$
\text { fi, L, W, tav, twal, rH2O, rtri, Bcal, Vg }
$$

$$
K=f(d, s 1, s 2, z 1, z 2, w)
$$

Then the configuration optimization is turned into the proper setting of array, diameter, rows and columns and the intervals of tubes. Hence, compatible combination of these factors above can minimize the area of heat transfer, maximize the heat transfer coefficient and guarantee the amount of heat transfer designed.

\subsection{Binary programs of GA and optimization analysis.}

According to the requirment of the design, several plans are brought up empirically by engineers. After the calculation, evaluation and analysis, the optimal plan is chosen as the final one. This kind of optimization, to a great extent, depends on the empiric experience of designers, with a low efficiency and the scope limited, hardly ensuring the current plan most suitible in this very case [3].

This section involves the programing in binary codes, implementing the functions of GA. Based on the program, array of the convection is optimized.GA programing in binary codes possesses the advantages: easy coding and decoding; feasible crossing and mututation feasible; compliane with the principle of the minimum character coding; convienent for the pattern theory for theoretical analysis and so on[4].The optimization procedure is introduced below:

1) Group estabishment

The number of the individuals in the group is 300; the maximum generation is 300 ; as the flow velocity of flue gas is determined by configuration, thus the number of determining factors is 5 , which can be changd into the optimization problems with five degrees of freedom; The gap between generations is set 0.9 ; the bits of the binary code is set 20 , then the describer of the scope is set, to limit the scope wherethe binary codes are coded into real values. The function crtbp from sheffield_gatbx is taken advantage of to create the initial group, and bs2rv turn these individuals into real values. Therefore, 300 plans with different parameters, which are randomly distributed are obtained

2) Objective function and constraints

Calculate the values of objective function of each individual, namely calculating the according heated surfaces of each plan. Since the parameters of individuals are randomly selected, there may exist the conditions of exceeding the size of fluepipe, deficient absorbed heat and too fast or slow flow velocity of flue gas. In order to eliminate these individuals in further generations, a set of penalty functions are written to restrict the relation among these parameters, including the constraints of crosswise and longitudinal sizes, the longitudinal rows of tubes, recommended ratio of interval and diameter, flow velocity of flue gas and absorbed heat. When the plan violates one (or more) of these contraints, its value of objective function will be added with a value from penalty function, to make it in an unfavourable position in further competition to accelarate the elimination.

\section{3) Selection}

After the introduction of penalty function adding to the objective function, the linear suitability is distributed to each individual based on the difference of values of objective function. After the calculation of suitability of each individual, the selection begins which follows the principle: the individuals with high values of suitability have great chance to be selected while the ones with relatively low values of suitability have great chance to be eliminated. The newly chosen group is called posterity whose number is determined by initial number and gap. 


\section{4) Cross and mutation}

The genes in binary codes of selected individuals will be swaped with each other in order to produce a better varity of group, where the possibility of swapping can be set. In this case, the value is set 0.7. After swaping genes, the inidividuals will be experiencing a period of mutation: in terms of binary code, 0 will be turned into 1 and vice versa with the aim to keep the group of better varity.

5) Objective function calculation and insertion

Individuals ,after the swaping and mutation period, will be asseted by calculating their values of objective function. Then the suitability will be distributed as (2) states. Then the posterity and initial group will be re-inserted based on the suitability to create a new group which shares the same amount with the former group.

6) Iteration

Repeat the procedure (2)-(5), and the optimization result is finally achieved.

Results from the program:

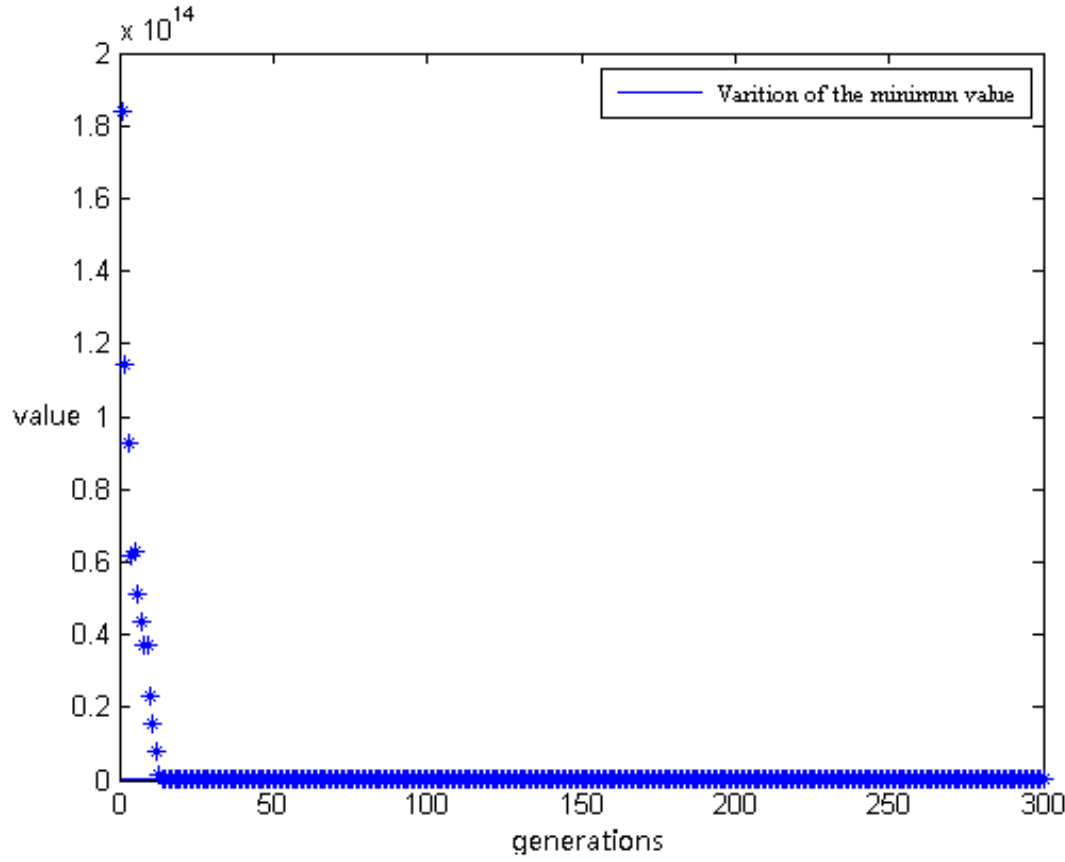

Fig. 4 Varition of the minimun value of objective function

The individuals convege to the parameters as following:

Diameter: $41.40 \mathrm{~mm}$, crosswise interval: $65.54 \mathrm{~mm}$, longitidinal interval $113.10 \mathrm{~mm}$, rows along the crosswise direction: 56.36, rows along the longitidinal interval direction, according area of surface: $496.84 \mathrm{~m}^{2}$, absorbed heat and average velocity of flue gas. The time for running the programs is 40 seconds.

Have these parameters round to nearest intergers, and redo the thermal calculation then we can have a comparison as shown in Table1. 
Table 1 Comparison of before and after optimization(group programed by binary codes)

\begin{tabular}{|c|c|c|c|c|c|c|}
\hline & $\begin{array}{l}\text { diameter } \\
\text { /mm }\end{array}$ & $\begin{array}{l}\text { rows along } \\
\text { the } \\
\text { crosswise } \\
\text { direction }\end{array}$ & $\begin{array}{l}\text { rows along } \\
\text { the } \\
\text { longitudinal } \\
\text { direction }\end{array}$ & $\begin{array}{c}\text { interval } \\
\text { along the } \\
\text { crosswise } \\
\text { direction/mm }\end{array}$ & $\begin{array}{l}\text { interval } \\
\text { along the } \\
\text { longitudinal } \\
\text { direction } \\
\text { /mm }\end{array}$ & $\begin{array}{c}\text { area of } \\
\text { surface } / \mathrm{m}^{2}\end{array}$ \\
\hline $\begin{array}{c}\text { results from } \\
\text { GA }\end{array}$ & 41.3984 & 56.357 & 9.843 & 65.535 & 113.098 & 396.8 \\
\hline $\begin{array}{l}\text { results } \\
\text { rounded }\end{array}$ & 42 & 56 & 10 & 65 & 113 & 406.38 \\
\hline \multirow[t]{2}{*}{$\begin{array}{c}\text { before } \\
\text { optimization }\end{array}$} & 51 & 40 & 14 & 100 & 120 & 468.8 \\
\hline & $\begin{array}{c}\text { inlet } \\
\text { temperature } \\
/^{\circ} \mathrm{C}\end{array}$ & $\begin{array}{c}\text { outlet } \\
\text { temperature } \\
/{ }^{\circ} \mathrm{C}\end{array}$ & $\begin{array}{l}\text { velocity of } \\
\text { flue gas } \mathrm{m} / \mathrm{s}\end{array}$ & $\begin{array}{c}\mathrm{K} \\
\mathrm{W} /\left(\mathrm{m}^{2} \times \mathrm{K}\right)\end{array}$ & $\begin{array}{c}\text { heat } \\
\text { absorbed } \\
\text { J/kg }\end{array}$ & $\begin{array}{c}\text { error in } \\
\text { heat } \\
\text { absorbed\% }\end{array}$ \\
\hline $\begin{array}{c}\text { results from } \\
\text { GA }\end{array}$ & 982.4 & 465 & 9.910 & 54.808 & 5940.1 & 1.31 \\
\hline $\begin{array}{l}\text { results } \\
\text { rounded }\end{array}$ & 982.4 & 473 & 8.51 & 52.96 & 5848.6 & 2.85 \\
\hline $\begin{array}{c}\text { before } \\
\text { optimization }\end{array}$ & 982.4 & 442.40 & 8.54 & 47.01 & 6019.2 & -- \\
\hline
\end{tabular}

It can be concluded that on the premise of keeping approximately constant heat absorbed, the rounded results can increase the $K$ by $10 \%$. Hence, it can effectively decrease the areas of the heated surface, economizing on the consumption of steel.

\subsection{The effect of constraints on results.}

Since the optimization is applied in specific boiler, the parameters configuration should be limited into proper range so that the size of furnace can not be interfered. The current solutions include: Design specific GA operator to make any solutions of GA are qualified to fit the furnace; the constraints are not considered in coding period, but in the criteria period to decide whether the solution is used or abandoned; Use penalty functions to deal with the constraints[7].

The present study takes advantage of the penalty function whose main idea is to add an extra value (penalty function) to these unqualified individual while calculating the objective functions, which will decrease the according suitability, making them have less chance to inherent their genes into next generation. The difficulty of the proper penalty function lies in that not only the should the contraints be measured, but also the calculation efficiency be considered.

1. The superme constraint - heat absorbed: The optimization is conducted under the condition with designed temperature of flue gas and working medium, namely fixed average temperature difference and heat absorbed. If the heat absorded varies to a great extent, the outlet flue gas temperature will vary greatly, resulting in great change in average temperature difference. Hence, in order to avoid deviating from the designed condition, this constraint should be controled strictly.

2. Less superme constrant-size of boiler: Since the optimization is conducted in specific boilers, the size of the array tube should in compliance with the size of the boiler, which indicates inference should be avoided. Otherwise, the configuration is not appliable optimization. 
3. Low contraint - recommended average velocity of flue gas: The recommended average velocity of flue gas in the Industrial Boiler Manual is from $8-10 \mathrm{~m} / \mathrm{s}$. The lower velocity will result in congestion of ash on the heated surface, decreasing the heat transfer coefficient, while significant pressure drop and tube's abrasion will occur with higher velocity.

4. Lower constraint - the recommended ratio of intervals along crosswise and longitidual direction and diameter of tube: This relationship is also included to get a better evolution result and converge to a optimal result.

The according codes are written in the programs to monitor the condition of overstep the constraints. The optimized plan is fine agreement with these contraints, indicating a satisfactory plan is achieved.

\section{Summary}

This present study first introduces the concept of Genetic Algorithm, features and procedures. Based on the conditions, the mathematical model of tubes' configuration has been established and the optimization procedures have been demonstrated in details: creation of group, calculation of objective function, distribution of suitability, selection, cross, mutation and insertion etc. Moreover, penalty function is brought up to limit the parameters. An optimal plan is obtained, and such method should be applied to economizer and air heater. It can also be taken as a reference for other optimization problem.

\section{Literature References}

[1] Haupt RL, Haupt SE. Practical genetic algorithms. 2nd Ed. John Wiley \& Sons; 2004.

[2] P. Talebizadeh, M.A. Mehrabian, M. Abdolzadeh, Prediction of the optimumslope and surface azimuth angles using the genetic algorithm, Energy andBuildings 43 (2011) 2998-3005.

[3] K. Deb, A. Pratap, S. Agarwal, T. Meyarivan, A fast and elitist multiobjectivegenetic algorithm: NSGA-II, IEEE Transactions on Evolutionary Computation 6(2002) 182-197.

[4] Selbas R, Kı zı lkan O, Reppich M. A new design approach for shell-and-tube heat exchangers using genetic algorithms from economic point of view. Chem Eng Process 2006;45:268 - 75.

[5] Ponce JM, Serna M, Rico V, Jimenez A. Optimal design of shell-and-tube heat exchangers using genetic algorithms. Comput Aid Chem Eng 2006;21:985 - 90.

[6] Ponce-Ortega JM, Serna-Gonzalez M, Jimenez-Gutierrez A. Use of genetic algorithms for the optimal design of shell-and-tube heat exchangers. Appl Therm Eng 2009;29:203 - 9.

[7] Sanaye S, Hajabdollahi H. Thermal-economic multi-objective optimization of plate fin heat exchanger using genetic algorithm. Appl Energy 2010;87:1893 - 902.

[8] Najafi H, Najafi B, Hoseinpoori P. Energy and cost optimization of a plate and fin heat exchanger using genetic algorithm. Appl Therm Eng 2011;31:1839-47.

[9] Hajabdollahi H, Ahmadi P, Dincer I. Thermo-economic optimization of a shell and tube condenser using both genetic algorithm and particle swarm. Int J Refrig 2011;34:1066 - 76.

[10] S. Rajasekaran, T. Kannadasan, optimization of shell and tube heat exchangers using modified genetic algorithm, Int. J. Control Autom. 3 (4) (December, 2010). 